\title{
Air Displacement Plethysmography and Resistance Exercise
}

Joan Cebrick Grossman

University of Scranton, joan.grossman@scranton.edu

Ronald W. Deitrick

University of Scranton

Follow this and additional works at: https://nsuworks.nova.edu/ijahsp

Part of the Internal Medicine Commons, and the Other Medical Specialties Commons

\section{Recommended Citation}

Grossman JC, Deitrick RW. Air Displacement Plethysmography and Resistance Exercise. The Internet Journal of Allied Health Sciences and Practice. 2015 Apr 01;13(2), Article 4.

This Manuscript is brought to you for free and open access by the College of Health Care Sciences at NSUWorks. It has been accepted for inclusion in Internet Journal of Allied Health Sciences and Practice by an authorized editor of NSUWorks. For more information, please contact nsuworks@nova.edu. 


\title{
Air Displacement Plethysmography and Resistance Exercise
}

\begin{abstract}
Purpose: The purpose of this study was to examine the effect of resistance exercise (RE) on body composition using air-displacement plethysmography (ADP) within 2 hours of completing RE. Methods: Thirteen university students ( 9 males and 4 females; $18.8 \pm 0.5 \mathrm{yrs}$; mean \pm SD) volunteered for this study. The RE program ( $55.4 \pm 8.7 \mathrm{~min}$ ) consisted of a combination of upper- and lower-body extremity exercises and abdominal exercises for a total of nine different exercises. Relative body fat (BF) was assessed $(1.76+0.4 \mathrm{hr})$ using ADP before and after the RE along with skinfold (SF) measures, body mass (BM) and respiratory quotient. Results: Statistically significant reductions in pre-post $R E$ relative $B F$ measurements $(p=0.036)$ were observed using ADP along with statistically significant reductions in body volume (BV) $(p=0.005)$, body mass $(B M)(p=0.038)$ and fat mass $(F M)(p=0.020)$. The mean intraclass correlation coefficients (ICC) for these variables was 0.99 . No significant pre-post differences in fat-free mass (FFM), body mass index (BMI) or skinfold (SF) values were reported. Conclusions: This study demonstrates the potential adverse effects of assessing relative BF within 2 hours after RE. Significant differences in prepost relative BF were supported by significant reductions in BV, BM, and FM. The results of this study support the manufacturer guidelines using ADP and RE restriction prior to body composition assessment.
\end{abstract}




\title{
IIJAHSP
}

\section{The Internet Journal of Allied Health Sciences and Practice}

Dedicated to allied health professional practice and education

Vol. 13 No. 2 ISSN 1540-580X

\section{Air Displacement Plethysmography and Resistance Exercise}

\author{
Joan Cebrick Grossman PhD, RD 1 \\ Ronald W. Deitrick, PhD, FACSM ${ }^{2}$
}

1. Assistant Professor, Exercise Science and Sport Department, University of Scranton, Scranton, PA

2. Associate Professor, Exercise Science and Sport Department, University of Scranton, Scranton, PA

United States

\begin{abstract}
Purpose: The purpose of this study was to examine the effect of resistance exercise (RE) on body composition using airdisplacement plethysmography (ADP) within 2 hours of completing RE. Methods: Thirteen university students (9 males and 4 females; $18.8 \pm 0.5 \mathrm{yrs}$; mean \pm SD) volunteered for this study. The RE program ( $55.4 \pm 8.7 \mathrm{~min})$ consisted of a combination of upper- and lower-body extremity exercises and abdominal exercises for a total of nine different exercises. Relative body fat (BF) was assessed $(1.76 \pm 0.4 \mathrm{hr})$ using ADP before and after the RE along with skinfold (SF) measures, body mass (BM) and respiratory quotient. Results: Statistically significant reductions in pre-post RE relative BF measurements $(p=0.036)$ were observed using ADP along with statistically significant reductions in body volume (BV) $(p=0.005)$, body mass $(B M)(p=0.038)$ and fat mass (FM) $(p=0.020)$. The mean intraclass correlation coefficients (ICC) for these variables was 0.99 . No significant pre-post differences in fat-free mass (FFM), body mass index (BMI) or skinfold (SF) values were reported. Conclusions: This study demonstrates the potential adverse effects of assessing relative BF within 2 hours after RE. Significant differences in pre-post relative $\mathrm{BF}$ were supported by significant reductions in $\mathrm{BV}, \mathrm{BM}$, and FM. The results of this study support the manufacturer guidelines using $\mathrm{ADP}$ and RE restriction prior to body composition assessment.
\end{abstract}

\section{INTRODUCTION}

Air-displacement plethysmography (ADP), commonly assessed using the BOD POD® Life Measurement Incorporated (LMI) (Concord, CA, USA), has become widely popular as a densitometry measure of body composition. ${ }^{1}$ The increased interest in body composition assessment has been in part due to the epidemic of obesity and the reduced technical requirement needed for testing purposes using the $B O D P O D \otimes$ when compared to hydrostatic weighing. ADP assessment is also less time-consuming and has been cross-validated with hydrostatic weighing. ${ }^{2}$ The basic principle of ADP involves the measurement of body volume (BV), which in turn is used to calculate body density (BD). The BOD POD® consists of a closed chamber of atmospheric air with a known volume. As an individual enters the BOD POD ${ }^{\circ}$ chamber for testing, an air-tight seal is formed with a new determined volume of air. The new air volume is then subtracted from the total volume of the chamber air to estimate the individual's volume from which $\mathrm{BD}$ and subsequent relative body fat $(\mathrm{BF})$ is estimated. ${ }^{1}$

The validity of body composition when using ADP has been investigated regarding the effects of body temperature and moisture, wherein an increase in body heat and moisture resulted in an underestimation of relative body fat. ${ }^{3}$ In addition, the effect of clothing type worn during ADP has been investigated, such that the entrapment of air under lose fitting clothing underestimates relative $\mathrm{BF}^{2}$ More recently, the effect of acute, moderate-intensity aerobic exercise on the accuracy of ADP has been studied, and the findings of this research support the recommended guidelines for testing with respect to prior aerobic exercise as relative $\mathrm{BF}$ was reduced with acute moderate-intensity exercise. ${ }^{4}$ However, to date, there are no peer-reviewed, empirical articles regarding the effects of resistance exercise (RE) on the assessment of body composition using ADP. Yet, the guidelines for assessing body composition using ADP specifically prohibit any exercise 2 hours prior to ADP assessment. The purpose of this study was to investigate the effects of RE exercise on body composition using ADP within 2 hours post-exercise to determine if relative $B F$ changes with $R E$. 


\section{METHODS}

The study protocol was reviewed and approved by the University of Scranton Institutional Human Subjects Review Board (IRB). Additionally, the procedures followed in the study adhered to the University of Scranton's IRB and the Health Insurance Portability and Accountability Act (HIPPA) guidelines.

\section{Study Population}

Thirteen college-aged University of Scranton students ( 9 males and 4 females) volunteered to participate this study (18.8 \pm 0.5 yrs), which was a repeated measures, case-controlled study, and there were no drop-outs. All subjects completed an informed consent form and Physical Activity Readiness Questionnaire (PAR-Q), prior to the start of their participation. ${ }^{5}$ A numerical code was assigned to each subject for data coding and management, ensuring subject anonymity and confidentiality.

\section{Measurements}

\section{Pre-Exercise Metabolic and Body Composition Measurements}

All subjects slept for a minimum of 6 hours and fasted for at least 8 hours. In order to ensure a fasted state, respiratory quotient was determined for each subject using the "canopy" method with the Vmax ${ }^{\top M}$ metabolic cart (San Diego, CA, USA). Body composition was also estimated via skinfold (SF) measurements using a Lange caliper (Ann Arbor, MI, USA). The SF measurements were based upon the equation from Durman \& Wormseley, which included four sites: biceps, triceps, subscapular, and suprailiac. 6 Manufacturer guidelines were followed and SF measurements were conducted on the right side of the participants' bodies. Three measurements were taken at each site and the average of the three measurements was used to determine each measurement site. Estimated relative BF, which was based upon gender and age, was then determined by the sum of the four measurement sites.

Height (cm) was initially determined using a standard stadiometer (Aryton, Model S100, Prior Lake, MN, USA). Body composition assessments using ADP was then measured and followed the LMI guidelines, except for the exercise recommendations, which indicated no exercise for at least 2 hours prior to testing. All subjects wore a Lycra® swim cap and had their hair tucked and compressed firmly underneath the cap with no apparent air pockets. Males wore form-fitting, single layer compression shorts or swim trunks and females wore either a jog bra and form-fitting leggings or a full swim suit. Each subject wore their same clothing for pre- and post-exercise measurements. Immediately prior to testing, all subjects voided their urine and removed all jewelry and glasses for the testing period. Quality control procedures and calibration of the BOD POD® were performed daily per LMI recommendations. The BOD POD® was calibrated each morning on the days testing occurred. The calibration procedures specifically followed the LMI protocol which began with a system warm-up for at least 30-minutes, ensuring optimal temperature for all electrical components. This was then followed by the analysis of the system hardware, a scale calibration, a scale check, and an autorun of the system along with a final volume performance calibration. Prior to the testing of each subject, a volume performance calibration and scale check were also completed. The ADP protocol involved 2 to $3, \sim 45$-second measurements, with the subject inside the BOD POD® chamber using estimated thoracic lung volumes. Body fat (\%BF), body mass (BM), fat mass (FM), fat-free mass (FFM), body volume (BV) and body mass index (BMI) were determined using ADP. Once the initial ADP measures were completed, the subjects began the RE program.

\section{Resistance Exercise Protocol}

All study participants completed an independent RE workout at the University of Scranton Musculoskeletal Laboratory. Each subject had strength trained for at least 3 months prior to volunteering for the study. The exercise routine consisted of abdominal exercises, "crunches", along with upper and lower body extremity resistance exercises that included leg press, leg extension, hamstring curl, latissimus dorsi pull-downs, incline bench press, shoulder press, biceps curls and triceps push-downs. The subjects used both cable-assisted machines and free weights for the RE workout. Each participant completed 3 sets of each exercise to failure. The subjects were instructed to remain in a fasted state, but encouraged to stay hydrated consuming only water, both during and after the completion of their exercise session. The average exercise time for the RE session was (55.4+8.8 min).

\section{Post-exercise Measurements}

The mean time the subjects returned to the laboratory was $(1.76+0.43$ hours $)$ post-exercise for repeated measurements, which included respiratory quotient to ensure a fasted state, along with body composition measurements using SF and ADP. All testing guidelines and protocols were followed as previously indicated. 


\section{Statistical Analysis}

SPSS Statistics 19.0 (SPSS, Chicago, IL, USA) was used for statistical analyses. Results are expressed as mean \pm standard deviation (SD). Paired samples t-tests were used to determine statistical differences between pre- and post-exercise measurements. An alpha value of 0.05 was used to signify statistical significance.

\section{RESULTS}

Thirteen, college-aged students ( $18.8 \pm 0.55 \mathrm{yrs})$ (9 males and 4 females) with a mean height of $(173.8 \pm 11.2 \mathrm{~cm})$ completed the pre- and post-exercise measures. The results revealed significant differences $(p<0.05)$ between pre- and post-exercise measurements, in \%BF using ADP along with BM, BV and FM, along with strong intraclass correlations (ICC), shown in Table 1.

\begin{tabular}{|l|c|c|c|c|}
\hline \multicolumn{7}{|c|}{ Table 1. Pre- and Post-Exercise Measures, Mean (+ Standard Deviation) } \\
\hline & Pre-Exercise & Post-Exercise & P-value & ICC \\
\hline$\%$ Body Fat (ADP) & $16.4 \pm 6.9$ & $15.7 \pm 7.1$ & 0.036 & 0.99 \\
\hline Body Mass (kg) & $70.7 \pm 10.7$ & $70.5 \pm 10.7$ & 0.038 & 1.00 \\
\hline Body Volume (kg/L) & $66.4 \pm 9.7$ & $66.1 \pm 9.6$ & 0.005 & 0.99 \\
\hline Fat Mass (kg) & $11.2 \pm 4.1$ & $10.7 \pm 4.3$ & 0.020 & 1.00 \\
\hline
\end{tabular}

Statistical significance $(\mathrm{p}<0.05)$

Study variables that were not statistically significant at $(p<0.05)$, included SF and FFM as indicated in Table 2.

Table 2. Pre- and Post-Exercise Measures, Mean (t Standard Deviation)

\begin{tabular}{|l|c|c|c|}
\hline & Pre-Exercise & Post-Exercise & P-Value \\
\hline Body Mass Index (BMI) & $23.3 \pm 2.8$ & $23.3 \pm 2.7$ & 0.056 \\
\hline 4 site Skinfold Sum (mm) & $53.3 \pm 15.5$ & $56.3 \pm 12.8$ & 0.113 \\
\hline Fat Free Mass (kg) & $60.0 \pm 12.3$ & $59.8 \pm 12.1$ & 0.648 \\
\hline
\end{tabular}

Statistical significance $(p<0.05)$

\section{DISCUSSION/CONCLUSION}

This study examined the effect of RE on body composition using ADP within 2 hours of completing RE. Results of the study indicated that \%BF, BM, BV and FM were all significantly lower following a RE session averaging (55.4_8.8min).

The differences seen are thought to be a result of the intervention, RE, and not a test/re-test influence. Multiple different studies over the past two decades have measured the test/re-test reliability of ADP using various sample sizes and ages along with trials performed within day and between days, males, females, lean, and overweight subjects, all of which have supported ADP as a reliable method of estimating body composition. . $^{-11}$ Vescovi et al. found the test/re-test reliability of \%BF was 0.99 , while Collins et al. reported the test/re-test reliability assessing \%BF was 0.994 with a technical measurement error of $0.448 \% .10,11$ Tucker et al. tested the reliability of the BOD POD® and the effect of multiple assessments had an absolute mean difference of .55 and ICC of .998 .7 Noreen and Lemon assessed body density and successfully measured the reliability of the BOD POD® with a large, heterogeneous sample, and the results indicated an ICC of $0.996 .{ }^{9}$ Within-day trials were observed to be more accurate; consequently such measures were followed for this study. ${ }^{8}$

Possible explanations for these differences may be a result of water loss through sweating which may result in fluid loss and a decrease in $\mathrm{BM}, \mathrm{BV}$, and $\mathrm{FM}$, ultimately decreasing \%BF. During RE, plasma volume loss is proportional to the intensity of the exercise, with possible transient fluid losses from the vascular space up to $15 \% .^{12}$ As exercise intensity increases, plasma volume losses may occur with the majority of fluid loss from the interstitial space. As the duration of the exercise continues, both the oncotic and osmotic pressures increase, resulting in more plasma movement out of the vascular compartment and into the interstitial space. With increased exercise intensity, fluid may also be lost from the intracellular compartment. Additionally, body hydration, whether dehydrated or hypo hydrated, has been shown to have an effect on other established methods of body composition such as SF measurements and bioelectrical impedance. ${ }^{13}$ It has been reported that as little as an approximated $2 \%$ decrease in body weight from dehydration resulted in a decreased body composition with ADP, which would support the importance of hydration status and accurate measures when measuring body composition with ADP. ${ }^{13}$ Subjects in this study were encouraged to stay hydrated during the testing period. However, they were also encouraged to complete 3 sets of RE to 
failure. Based upon the possible shifting of plasma volume due to exercise intensity, a decrease in plasma volume may be a contributor to a decrease in BM, BV, FM, and ultimately \%BF. The technique of ADP assumes the ratios of water, protein, and mineral content of all adults are the same with differences only in fat mass. ${ }^{14}$ It is highly unlikely that fluid loss would be the same for different persons. Although there is no known relationship between acute sweat loss due to exercise and decreased ADP measurements, it is logical that dehydration due to exercise and fluid loss would decrease both BM and BV, which is consistent with similar work. 3,4,14

There were some limitations of the present study, which included a small sample size and no control group. A larger sample with a control may provide greater validation. Another limitation was that the majority of the subjects were Caucasian; thus, the result may not be generalizable to other racial and ethnic groups. Hydration status was not measured, which can affect BM and BV measures. The measurement of pre- and post-exercise hydration via specific gravity of urine is a simple test and suggestion for future work to determine if the decreases noted in \%BF, BM, BV, and FM within this work could possibly be related to hydration status.

In conclusion, the results of this study encourages and supports the manufacturer guidelines, such that body composition measurements using ADP taken prior to the recommended 2 hours post-exercise time frame resulted in a statistically significant reduction in \%BF, BM, BV, and FM. It is therefore recommended to follow the manufacturer guidelines when measuring body composition using ADP as the accuracy of the body composition measurements may be compromised.

\section{ACKNOWLEDGEMENTS}

We would like to thank the students at the University of Scranton who participated in this study and Drs. Paul Cutrufello and Thomas Hogan for their statistical reviews.

\section{REFERENCES}

1. BOD POD body composition tracking system operator's manual 2004, Life Measurement Inc., Concord.

2. Thompson DL, King GA, Evans MJ, Moreau K, McLaughlin JE, Fulkerson B. Effect of clothing type on bod pod (R) measurement of body fat: comparison to hydrostatic weighing. Med Sci Sports Exerc. 1999;31(5):S203.

3. Fields DA, Higgins PB, Hunter GR. Assessment of body composition by air-displacement plethysmography: influence of body temperature and moisture. Dyn Med. 2004;3:1-7. [PMID 15059287]

4. Otterstetter R, Johnson KE, Kiger DL, Agnor SE, Edwards J, Naylor JB, et al. The effect of acute moderate-intensity exercise on the accuracy of air-displacement plethysmography in young adults. Eur Jour Clin Nut. 2013;67: 1092-4. [PMID 23859994]

5. Canadian Society for Exercise Physiology; Health Canada. Physical activity readiness questionnaire; Par-q \& you. Minister of Public Works and Government Services Canada 2002:1-2.

6. Durman JVGA, and Womersley J. Body fat assessed from total body density and its estimation from skinfold thickness. Measurements on 481 men and women aged from 16 to 72 years. Br J Nutr. 1974;32:779.

7. Tucker LA, Lecheminant JD, Bailey BW. Test-retest reliability of the Bod Pod: the effect of multiple assessments. Percept Mot Skills. 2014;118(2):563-70. [PMID 24897887]

8. Anderson, DE. Reliability of air displacement plethysmography. J Strength Cond Res. 2007;21(1):169-72. [PMID 17313287]

9. Noreen EE, Lemon PW. Reliability of air displacement plethysmography in a large, heterogeneous sample. Med Sci Sports Exerc. 2006;38(8):1505-9. [PMID 16888466]

10. Vescovi JD, Zimmerman SL, Miller WC, Hildebrandt L, Hammer RL, Fernhall B. Evaluation of the BOD POD for estimating percentage body fat in a heterogeneous group of adult humans. Eur J Appl Physiol. 2001;85(3-4):326-32. [PMID 11560087]

11. Collins MA, Millard-Stafford ML, Sparling PB, Snow TK, Rosskopf LB, Webb SA, Omer J. Evaluation of the BOD POD for assessing body fat in collegiate football players. Med Sci Sports Exerc.1999;31(9):1350-6. [PMID 10487380]

12. Wilmore JH, Costill DL, Kenney WL. Physiology of Sport and Exercise, 5th edn. Human Kinetics: Champaign, Illinois, 2008, pp 193, 359-60.

13. Drummond MJ, Parcell AC. Air displacement plethysmography under dehydrated and hyperhydrated conditions. Med Sci Sports Exerc. 2004;36:S72.

14. Vukovich MD, Peeters BM. Reliability of air-displacement plethysmography in detecting body composition changes after water ingestion and after creatine supplementation. J Exerc Phys. 2003;6:115-22.

(C) The Internet Journal of Allied Health Sciences and Practice, 2015 